\title{
„Die Kinderradiologie muss die Entwicklungen der modernen Bildgebung mitgehen!“
}

\author{
Interview mit Univ.-Professorin Dr. med. Gundula Staatz, Kongresspräsidentin des \\ 100. Deutschen Röntgenkongresses und Leiterin der Sektion Kinderradiologie, Klinik und \\ Poliklinik für Diagnostische und Interventionelle Radiologie, Universitätsmedizin Mainz
}

Zum 100. Mal findet vom 29. Mai bis 1. Juni 2019 in Leipzig der Röntgenkongress der Deutschen Röntgengesellschaft (DRG) statt. Zu den 3 Kongresspräsidenten gehört Univ.-Professorin Dr. med. Gundula Staatz, Leiterin der Sektion Kinderradiologie, Klinik und Poliklinik für Diagnostische und Interventionelle Radiologie der Universitätsmedizin Mainz. Im Interview spricht die erste Kongresspräsidentin der DRG über das Kongress-Thema „Jung und Alt" und Highlight-Sitzungen aus dem Bereich der Kinderradiologie. In einem wissenschaftlichen Übersichtsartikel präsentiert Prof. Staatz dazu gemeinsam mit renommierten nationalen und internationalen Kolleginnen und Kollegen das Bild einer zukunftsfähigen, hochspezialisierten Kinderradiologie. Zudem wird in der Rubrik „Der interessante Fall“ anhand zweier Fallbeispiele beleuchtet, warum Tuberkulose aktuell eine Herausforderung für die Radiologie darstellt.

Was bedeutet es für Sie, als Kongresspräsidentin des 100. Deutschen Röntgenkongresses zu amtieren?

Für mich ist es eine große Ehre und Auszeichnung, dass ich als erste Frau das Kongresspräsidenten-Amt innehaben und zudem die Kinderradiologie auf diesem besonderen Kongress vertreten darf. Das ist natürlich auch eine Würdigung meines Fachgebietes, über die ich mich sehr freue.

Welche Veranstaltungen sollten die Teilnehmer beim 100. Deutschen Röntgenkongress auf keinen Fall versäumen?

Wir haben auf dem Röntgenkongress ein breit gefächertes wissenschaftliches und interessantes Fortbildungsprogramm zusammengestellt. Jeder Besucher, jede Besucherin kann sich aus dem Programm etwas herauspicken, das zu den Interessen passt. Der Kongress bietet aber auch eine gute Gelegenheit, über den Tellerrand hinaus zu blicken und Veranstaltungen zu besuchen, die andere fachliche Schwerpunkte thematisieren. Ich persönlich finde die Highlight-Sitzungen zu den 3 Hauptthemen des Röntgenkongresses wichtig, also Radiologie 4.0, Früherkennung und Jung und Alt. Zudem haben wir in diesem Jahr eine neue Highlight-Reihe Zukunft der Radiologie konzipiert, in welcher die Arbeitsgemeinschaften der DRG spannende Vorträge zur Entwicklung der Radiologie zusammengestellt haben. Und natürlich würden wir uns freuen, wenn möglichst viele Kongressbesucherinnen und -besucher zur Eröffnungsveranstaltung und zur Röntgen-Vorlesung kommen. Die Eröffnungsveranstaltung mit dem bekannten TV-Wissenschaftsjournalisten Ranga Yogeshwar und die RöntgenVorlesung mit dem Spiegel-Kolumnisten Sascha Lobo stehen ganz im Zeichen von künstlicher Intelligenz.

Sie haben als Kongresspräsidentin und Kinderradiologin den Schwerpunkt ,Jung und Alt" gewählt. Welche Inhalte werden dazu auf dem Kongress vertieft?

Wir haben diverse kinderradiologische Themen zur speziellen Bildgebung bei Kindern und Jugendlichen vorbereitet. Das betrifft Refresher-Kurse zur onkologischen, neonatologischen und muskuloskelettalen Bildgebung, aber auch fallbasierte Vorträge zur Bildgebung in der Sport-, Unfall- und Notfallmedizin. Als Kongresspräsidentin hatte ich die Gelegenheit, zum ersten Mal eigene Highlight-Sitzungen für die Kinderradiologie mitzugestalten - ich freue mich auf exzellente Vorträge von nationalen und internationalen Referentinnen und Referenten zu diesem Gebiet.

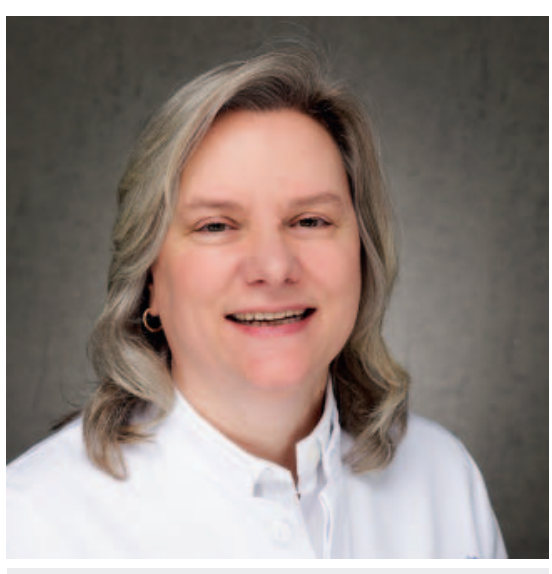

Univ.-Professorin Dr. med. Gundula Staatz

Können Sie eine dieser Highlight-Sitzungen näher beschreiben?

Am Kongress-Donnerstag nimmt die Sitzung Kinderradiologie 2025 - quo vadis? die Zukunft in den Blick. Darin geht es um die Entwicklung der Kinderradiologie vom Röntgen zum PET-MRT. Wir werden einen Vortrag zu zukunftsweisenden MR-Techniken hören und beleuchten, wie gering die Dosis beim Low-Dose-CT sein kann. Das ist ja ein ganz zentrales Thema, wenn Computertomografie für Kinder notwendig wird: Wie kann ich mit möglichst wenig Dosis die volle Bildinformation erzielen?

Neben der Bildgebung bei z. B. Lungenkrankheiten oder chronischen Erkrankungen wie der Glasknochenkrankheit haben es Kinderradiologen auch mit Kindesmisshandlungen zu tun. Wird es dazu auch eine Sitzung auf dem Kongress geben?

Wir haben am Kongress-Samstag eine Sitzung zum Thema Kindesmisshandlung, bei der die ZNS-Läsionsmuster, Skelettveränderungen oder auch Charakteristika von abdominellen Verletzungen behandelt werden. Das Thema Kindesmisshandlung ist wichtig. Wir sehen ca. ein Dutzend Patienten pro Jahr, aber die Dunkelziffer liegt ja viel, viel höher. Wir Radiologen sollten deshalb sensibilisiert sein, das schwierige 
Thema Kindesmisshandlung stets mitzudenken. Wichtig ist, dass die Betreuung dieser Patienten dann im Rahmen von sogenannten Kinderschutzgruppen, wie bei uns an der Universitätsmedizin Mainz, abläuft: Ein interdisziplinäres Team aus Mitarbeitern und Mitarbeiterinnen der Kinderklinik, Rechtsmedizin, Kinderradiologie, Kinderchirurgie, Sozialdienst u. a. bespricht die Anamnese, die Klinik und die Bildgebung gemeinsam und legt im Team dann das weitere Vorgehen fest. Voraussetzung ist aber natürlich, entsprechende Zeichen in der Bildgebung überhaupt erst einmal zu erkennen, um weitere Schritte einleiten zu können.

\section{Was ist das Besondere an Ihrem Beruf als Kinderradiologin innerhalb der Radiolo- gie?}

Wir decken eine enorme Altersspanne $a b$, vom kleinsten Frühgeborenen bis zu groBen Kindern und jungen erwachsenen Patienten, deren Erkrankungen, wie z.B die Mukoviszidose oder auch Stoffwechselerkrankungen, in der Kindheit beginnen und bis zum Erwachsenenalter gehen. Insofern ist Kinderradiologie Kinder- und Jugendmedizin, aber teilweise auch Erwachsenenmedizin. Patienten, die wir als Kinder sehen, betreuen wir häufig bis weit ins Erwachsenenalter hinein. Zudem haben wir ein anderes Krankheitsspektrum in der Kinderradiologie. Prinzipiell gilt: Kinder sind keine kleinen Erwachsenen. Dadurch ist das Tätigkeitsgebiet sehr anspruchsvoll, aber auch breit gefächert: Es reicht von der allgemeinen Kinder- und Jugendmedizin bis hin zu allen möglichen pädiatrischen Subspezialitäten, wie z. B. der Kinderonkologie, Kinderpulmonologie, Neonatologie, Kinderorthopädie, Kinderchirurgie oder Neuropädiatrie. Das macht die ganze Sache sehr spannend, weil abwechslungsreich. Was für mich persönlich immer ein Highlight der Kinderradiologie darstellt: Wir sind Allrounder bezüglich der verschiedenen bildgebenden Verfahren. Fast alle Kinderradiologen und Kinderradiologinnen in Deutschland wie auch international führen alle Verfahren durch, vom Ultraschall über konventionelle Röntgenuntersuchungen bis zu CT und MRT. Dabei steht für uns der Patient im Mittelpunkt - je nach Erkrankung wenden wir die Bildgebungsmethoden an, die für den
Patienten optimal sind und bei denen wir mit möglichst wenig Strahleneinsatz die bestmögliche Diagnose erzielen. Bei einem Kind steht fraglos der Ultraschall an erster Stelle, aber wenn das Kind darüber hinaus noch weitere Diagnostik benötigt, schicken wir es nicht woanders hin, sondern betreuen den kleinen Patienten von der ersten Untersuchung bis zur weiterführenden Schnitt-Bildgebung.

\section{Inwieweit erhalten angehende Kinderra- diologen Unterstützung, um in diesem anspruchsvollen Tätigkeitsfeld gut zu- recht zu kommen?}

Wir legen in der Kinderradiologie sehr viel Wert darauf, die Weiterbildungsassistenten und -assistentinnen behutsam an das Fachgebiet heranzuführen. Natürlich haben wir auch schwer kranke Kinder als Patienten und sehr mitgenommene Eltern, die ja immer mit dabei sind. Da braucht es Sensibilität, Einfühlungsvermögen und auch Geduld, um mit diesen Patienten und ihren Eltern adäquat umzugehen. In der Universitätsmedizin Mainz fangen die kinderradiologisch auszubildenen Ärzte und Ärztinnen immer mit der Diagnostik bei den größeren Kindern an, bevor wir zu den kleineren Kindern übergehen. Und wir lassen niemanden mit einem kranken Kind oder Jugendlichen allein, es ist immer ein kinderradiologischer Oberarzt bzw. ich selbst mit dabei, um die Untersuchungen zu überwachen.

Der Deutsche Ärztetag 2018 hat nach 5-jähriger, teilweise kontroverser Diskussion die neue (Muster-) Weiterbildungsordnung (MWBO) verabschiedet. Zu den Eckpunkten zählt der Erhalt der Schwerpunkte Neuro- und Kinderradiologie mit jetzt 2-jähriger Weiterbildungszeit. Wie schätzen Sie dieses Ergebnis ein?

Es gab Bestrebungen, die Schwerpunktfächer in den medizinischen Disziplinen insgesamt zu reduzieren. Daher sind wir sehr froh, dass die Kinderradiologie als Schwerpunktfach - genau wie die Neuroradiologie - erhalten geblieben ist. Die Weiterbildungszeit ist verkürzt worden, sie betrug früher 3 Jahre. Jetzt macht man eine Ausbildung zum Radiologen und setzt anschließend die Schwerpunktbezeichnung Kinderradiologie in einer 2-jährigen Wei- terbildungszeit oben drauf. Die Verkürzung der Weiterbildungszeit von 3 auf 2 Jahre zielt eindeutig darauf ab, mehr Nachwuchs für die Kinderradiologie zu gewinnen.

\section{Wie wird sich die Kinderradiologie zu- künftig verändern?}

Die Kinderradiologie wird auch zukünftig ein ganz modernes Fachgebiet bleiben, das spiegelt sich in der kinderradiologischen Anwendung von diversen Bildgebungsmethoden wider, wie es auf dem 100. Deutschen Röntgenkongress präsentiert wird. Aber die wenigsten modernen Bildgebungstechniken, die die Diagnostik erleichtern oder sogar verbessern, werden für Kinder entwickelt. Im Vordergrund steht die Anwendung der jeweiligen Bildgebungsmethode bei erwachsenen $\mathrm{Pa}$ tienten. Die Kinderradiologie muss diese neuen Bildgebungstechniken für Kinder und Jugendliche adaptieren, im Endeffekt an der modernen Bildgebung wachsen und ihre Entwicklungen mitgehen: Ob es sich um moderne Ultraschalltechniken handelt, Stichwort Elastografie, moderne Techniken zur Dosisreduktion in der Computertomografie oder hochspezialisierte Sequenztechniken in der Kernspintomografie, um zum Beispiel Bewegungs- und Atemartefakte zu unterdrücken, den Kontrast zu erhöhen oder zum Beispiel mittels Diffusions-Bildgebung möglichst wenig Kontrastmittel einsetzen zu müssen. Das gilt auch für die künstliche Intelligenz (KI), die aus meiner Sicht eine Chance bietet und keine Bedrohung darstellt. Wir haben es mit immer größeren Bilddatensätzen zu tun, mit Hunderten von Bildern. Wenn uns in dieser Situation ein Computer bei der Befundung hilft, ist das eine sehr gute Entwicklung. Natürlich muss letztlich der Radiologe bzw. der Kinderradiologe entscheiden, was ein Artefakt und was diagnoserelevant ist. Trotz dieses enormen Potenzials werden m. E. die KI-Systeme den Arzt in seinem Verhältnis zum Patienten nicht ersetzen können. Wenn ein Bildgebungssystem uns beispielsweise sagt, da ist nichts Besonderes zu sehen, ich sehe aber dem Kind an, dass es krank ist, dann gebe ich mich nicht mit einer unauffälligen, von künstlicher Intelligenz gesteuerten Bildgebung zufrieden. Ich weiß dann, dass ich noch einmal genauer 
hinschauen und vielleicht noch ein anderes Bildgebungsverfahren hinzunehmen muss, etwa ein MRT. Also, bei aller künstlichen Intelligenz - ohne Patientenkontakt und ohne den klinischen Blick geht es für mich aktuell und auch in absehbarer Zeit nicht.

Sie sind die erste Kongresspräsidentin seit dem ersten Deutschen Röntgenkongress 1905. Haben Sie einen Rat für junge Ärztinnen, die eine Karriere in der Radiologie anstreben?

Ich kann nur raten, zielgerichtet, engagiert und fleißig den angestrebten Weg zu gehen und sich für das Fachgebiet oder Spezialgebiet zu entscheiden, das einem am meisten Spaß macht - das ist für mich das Wichtigste. Für Ärztinnen gibt es mittlerweile gute Förderprogramme, wie z. B. Mentoring-Programme, an fast allen Universitäten. Für die nachfolgende Generation sollte es auch wichtig sein, sich berufspolitisch und für das Fachgebiet, das einem am Herzen liegt, zu engagieren. Denn wenn man nicht aktiv mitarbeitet, kann man auch nichts bewegen. Gerade weil es nicht so viele Kinderradiologen beziehungsweise Kinderradiologinnen gibt, kann ich nur jeden ermutigen, mitzuhelfen und mitzuarbeiten - auch wenn das zusätzliche Arbeit außerhalb der normalen Tagesroutine bedeutet -, um unser Fachgebiet voranzubringen.
Jetzt haben wir viel über Kinderradiologie gesprochen. Was erwartet die Kongressbesucher denn unter dem Stichwort „Alt"?

In der neonatologischen Kindermedizin werden die Kinder immer früher und damit kleiner geboren, heute haben wir es teilweise mit 300-Gramm-Frühchen zu tun. Auf der anderen Seite werden die Menschen immer älter, das Durchschnittslebensalter steigt weiter an. Auf dem Kongress werden daher Erkrankungen der Altersmedizin wie z.B der Morbus Alzheimer diskutiert.

Und was ist lhr persönliches Highlight auf dem 100. Deutschen Röntgenkongress?

Ich freue mich schon sehr auf die Eröffnungsveranstaltung und die Röntgen-Vorlesung, aber persönlich bin ich besonders gespannt auf die neue Highlight-Sitzung zur Reise- und Tropenmedizin. Mit steigender Reisefreudigkeit, auch in die entferntesten Länder dieser Welt, kehren die Menschen auch mit seltenen tropischen oder parasitären Erkrankungen zurück, mit denen wir es dann zu tun haben. Oder das Thema Tuberkulose: Die Erkrankung war in unserer Industriegesellschaft praktisch fast schon in Vergessenheit geraten, und erst mit der Zuwanderung aus den Nicht-Industrieländern ist die Tuberkulose jetzt wieder aktuell - darüber werden wir in dieser Highlight-Sitzung fallbasiert sprechen.

Also: Kommen Sie nach Leipzig, erleben und feiern Sie mit uns diesen einzigartigen Jubiläumskongress. Einen 100. Deutschen Röntgenkongress gibt es nur einmal! Wir haben alles getan und tun weiterhin alles dafür, dass es ein interessanter und erfolgreicher Kongress wird. Wir freuen uns darauf, die Teilnehmerinnen und Teilnehmer in Leipzig begrüßen zu dürfen!
Der 100. Deutsche Röntgenkongress findet vom 29. Mai bis 1. Juni 2019 im Congress Center Leipzig statt. Weitere Informationen unter https:// www.roentgenkongress.de.

Univ.-Professorin Dr. med. Gundula Staatz, Kongresspräsidentin des 100. Deutschen Röntgenkongresses, spricht im Kurzfilm über die Zukunft der Kinderradiologie und das Kongress-Thema „Jung und Alt“. Den Film finden Sie online unter https:// youtu.be/1k0jE7hNong

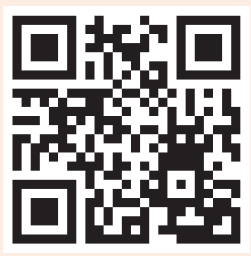

Einen Kurzfilm mit dem Kongresspräsidenten Professor Dr. med. Walter Heindel finden Sie online unter https://youtu.be/f_jzhOnO7h0. In diesem geht es um Früherkennung und ihre wachsende Bedeutung in der medizinischen Bildgebung.

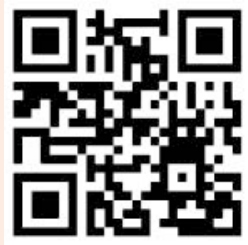

Professor Dr. med. Michael Forsting, ebenfalls Kongresspräsident des 100. Deutschen Röntgenkongresses, spricht im Kurzfilm über das Kongress-Thema „Radiologie 4.0“ und die Bedeutung der Digitalisierung für das Fach. Den Film finden Sie online unter https://youtu.be/ FPQRFCRM5Jg.

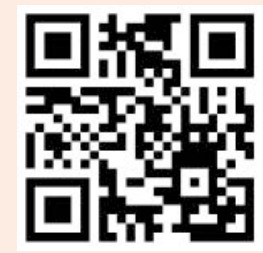

